\title{
A NUMERICAL METHOD FOR EVALUATING ZEROS OF SOLUTIONS OF SECOND-ORDER LINEAR DIFFERENTIAL EQUATIONS
}

\author{
RENATO SPIGLER AND MARCO VIANELLO
}

\begin{abstract}
A numerical algorithm for computing real zeros of solutions of 2 ndorder linear differential equations $y^{\prime \prime}+q(x) y=0$ in the oscillatory case on a half line is studied. The method applies to the class $q(x)=a+b / x+O\left(x^{-p}\right)$, with $a>0, b \in \mathbf{R}, p>1$.

This procedure is based on a certain nonlinear 3rd-order equation (Kummer's equation) which plays a role in the theory of transformations of 2 nd-order differential equations into each other, and was earlier introduced by F. W. J. Olver in 1950 to compute zeros of cylinder functions. A rigorous asymptotic and numerical analysis is developed by combining Boruivka's approach to the study of Kummer's equation and Olver's original idea. Numerical examples are presented.
\end{abstract}

\section{INTRODUCTION}

In this paper we develop a numerical algorithm for computing real zeros of solutions of 2 nd-order linear ordinary differential equations, such as

$$
y^{\prime \prime}+q(x) y=0,
$$

on a half line, in the oscillatory case.

Our method applies to the case

$$
q(x)=a+b / x+O\left(x^{-p}\right),
$$

with $a>0, b \in \mathbf{R}, p>1$, and the (technical) hypothesis of analyticity. The form of $q(x)$ in (1.2) includes many important cases, such as that of the Bessel equation, the Coulomb wave equation, several cases of the confluent hypergeometric equation (see [13]), etc.

The procedure is based on a certain nonlinear 3rd-order differential equation (Kummer's equation), which plays a role in the theory of transformations of linear ordinary 2 nd-order differential equations into each other, and was earlier introduced by F. W. J. Olver in 1950 [8] to compute zeros of cylinder functions.

Received July 31, 1989; revised November 6, 1989.

1980 Mathematics Subject Classification (1985 Revision). Primary 65L99, 34E20; Secondary $65 \mathrm{D} 20$.

Key words and phrases. Ordinary differential equations, zeros of functions, asymptotic and numerical approximation of zeros, special functions. 
Neither a general theory nor a proof of convergence was given by Olver at that time, but computational work was successfully carried out with the help of a certain algorithm suggested by the asymptotic behavior of the zeros.

In this paper, a more rigorous analysis is developed by combining Borůvka's approach to the study of Kummer's equation [4] and Olver's original idea. More precisely, we obtain an iterative scheme which converges in a suitable sense when $n$ (the iteration number) goes to $\infty$. Initialization is provided by a certain preliminary asymptotic analysis based on the asymptotic behavior of $q(x)$ in (1.1). Theorem 3.2 contains the main result of the paper (the proof of convergence), Corollary 3.3 shows that our method yields results much more accurate than the asymptotics alone when computing large zeros. At the same time, the theorem shows that the number of iterations needed for obtaining a given accuracy, say $\varepsilon$, is asymptotically proportional to $-\log \varepsilon$, as $\varepsilon \rightarrow 0^{+}$.

The plan of the paper is as follows. In $\S 2$, the problem is formulated and the relevant definitions and assumptions are laid down. The asymptotic part is worked out here, starting from the asymptotic representation assumed for $q(x)$. In $\S 3$, we give the main result, and a basic preliminary lemma is proved concerning a certain complex-valued function of three complex variables. In $\S 4$, several numerical examples are presented.

\section{Preliminary asymptotic anAlysis}

We are concerned with the problem of evaluating the real zeros of any particular solution of a 2 nd-order differential equation such as (1.1) on a half line, in the case when there exist infinitely many real zeros (oscillatory case).

Following Borůvka's terminology, we shall below refer to $q(x)$ in (1.1) as "the carrier" of the equation. For clarity and convenience, we shall consider first the case $b=0$ in (1.2). Results, similar to those in Theorem 3.2, for the general case $b \neq 0$ will be stated at the end of $\S 3$, in Remark 3.4.

We shall assume that the carrier enjoys the following properties. First of all, $q(x)$ is the restriction to the real half line, say $x>\rho$, of a function $q(z)$ holomorphic in an annular sector $S_{\rho, \gamma}$,

$$
S_{\rho, \gamma} \equiv\{z: z \in \mathbf{C},|z|>\rho,|\arg (z)|<\gamma\},
$$

for certain $\rho, \gamma$, where $\rho \geq 0$ and $0<\gamma \leq \pi / 2$. Moreover, we assume that $q(z)$ possesses the asymptotic structure

$$
\begin{array}{cc}
q(z)=a+Q(z), & a>0 \text { (constant), } \\
Q(z)=O\left(z^{-p}\right), & p>1 \text { (constant). }
\end{array}
$$

More precisely, we stipulate that the holomorphic function $Q(z)$ is estimated according to $(2.3)$ by

$$
|Q(z)| \leq K|z|^{-p}, \quad z \in S_{\rho, \gamma} .
$$

Our method for evaluating zeros is based on the derivation of a "phase function", $\alpha(x)$, relative to a given basis. Such a function is defined as any 
$C^{3}$-solution to the equation

$$
\tan \alpha(x)=u(x) / v(x),
$$

$u(x)$ and $v(x)$ being any two linearly independent solutions to (1.1); cf. [4, pp. 36-39]. In fact, the zeros being sought are the solutions to

$$
y(x, \alpha) \equiv u(x) \cos \alpha-v(x) \sin \alpha=0,
$$

which can be considered as a relation between $x$ and $\alpha$. A phase function has the property that

$$
\left|\alpha\left(x_{k}\right)-\alpha\left(x_{k-1}\right)\right|=\pi, \quad k=1,2,3, \ldots,
$$

for any two consecutive zeros of any particular solution to (1.1), although $\alpha$ depends on the special choice of the basis $(u(x), v(x))$; cf. [4, p. 38]. By inverting relation (2.7) one can thus compute the zeros, $x_{k}$.

On the other hand, we obtain from (2.5)

$$
\alpha^{\prime}(x)=-\frac{W}{u^{2}(x)+v^{2}(x)},
$$

where $W$ is the (constant) Wronskian of $u(x), v(x)$.

Equation (2.8) can be used to obtain $\alpha(x)$, whenever $u$ and $v$ are at hand; cf., e.g., [11]. Moreover, one can obtain at once $u^{2}(x)+v^{2}(x)$ as a solution to a well-known third-order linear differential equation that can be constructed explicitly $[2,3,12]$. It is possible, however, to eliminate $u$ and $v$ in (2.8), obtaining a closed-form third-order nonlinear differential equation satisfied by $\alpha(x)$ alone. This task can be accomplished by repeated differentiation and using the fact that $u^{\prime \prime}=-q u, v^{\prime \prime}=-q v$; the details are left to the reader. The resulting equation is

$$
\alpha^{\prime 2}=q(x)-\frac{1}{2}\{\alpha, x\}
$$

where

$$
\{\alpha, x\} \equiv \frac{\alpha^{\prime \prime \prime}}{\alpha^{\prime}}-\frac{3}{2}\left(\frac{\alpha^{\prime \prime}}{\alpha^{\prime}}\right)^{2}
$$

denotes the so-called "Schwarzian derivative" (cf., e.g., [6, p. 647]). Equation (2.9) is a special case of the so-called Kummer's equation, and appears in the theory of transformations of second-order linear differential equations; cf. [4]. One of the advantages in this approach is that one avoids evaluating the solutions $u, v$ to $(1.1)$ or their combination $u^{2}+v^{2}$.

The concept of phase plays a central role in describing solutions and properties of second-order linear differential equations. This provides, in fact, a key to obtaining any particular solution to $(1.1)$ (cf. $[4, \S 5.7$, p. 39]) as well as its zeros and its stationary points (see Remark 4.2 below). Approximating a certain phase by our procedure described below will enable us to compute globally the zeros of any particular solution. 
In [8] Olver derived a third-order nonlinear differential equation satisfied by the generic zero, $x=\rho(\alpha)$, of $y(x, \alpha)$ defined in (2.6), as a function of the real parameter $\alpha$. This equation can be written as

$$
\rho^{\prime 2}=\frac{1}{q(\rho)}\left(1-\frac{1}{2}\{\rho, \alpha\}\right) .
$$

It is easily checked that equation (2.9) changes into (2.11) upon the transformation $\rho=\rho(\alpha)$ and conversely (note that $\alpha$ is invertible as $\operatorname{sgn} \alpha^{\prime}=-\operatorname{sgn} W$; cf. (2.8)).

In [8] Olver was mainly concerned with the evaluation of zeros of cylinder functions. In this case it turns out that $\{\rho, \alpha\}=O\left(\alpha^{-3}\right)$ as $\alpha \rightarrow+\infty$, and he used the following iterative scheme:

$$
\begin{aligned}
\rho_{0}^{\prime 2} & =\frac{1}{q\left(\rho_{0}\right)}, \\
\rho_{n+1}^{\prime 2} & =\frac{1}{q\left(\rho_{n+1}\right)}\left(1-\frac{1}{2}\left\{\rho_{n}, \alpha\right\}\right), \quad n=0,1,2, \ldots,
\end{aligned}
$$

suggested by (2.11). We found it more convenient to base our approach on equation (2.9) rather than (2.11). Therefore, we introduce the iterative scheme

$$
\begin{aligned}
\alpha_{0}^{\prime 2} & =q(x), \\
\alpha_{n+1}^{\prime 2} & =q(x)-\frac{1}{2}\left\{\alpha_{n}, x\right\}, \quad n=0,1,2, \ldots .
\end{aligned}
$$

Note that Olver's method requires solving a differential equation at each step (for $\rho_{n+1}(\alpha)$ ), while ours merely involves quadratures (indeed, only one at the end of the whole procedure; see $\S 4$ ). We must however obtain the zeros from $\alpha_{n}(\cdot)$ while Olver's approach yields them directly. Moreover, no proof of convergence of the algorithm (2.12) was given in [8].

In $\S 3$, we shall prove that the sequence $\alpha_{n}^{\prime}(x)$ defined by (2.13), with $q(x)$ in the class described above, converges (in a suitable sense) to a solution, $\alpha^{\prime}(x)$, of (2.9) possessing a certain asymptotic behavior. This solution, $\alpha^{\prime}$, is such that relation (2.8) holds, $(u, v)$ being a Liouville-Green basis (or WKBJ; cf. [10]), and $W$ the (constant) Wronskian of $u$ and $v$. The initialization in (2.13) is suggested by the fact that we want to approximate precisely such a phase function. We call this phase function a "Liouville-Green phase".

If we set

$$
\phi_{n} \equiv\left(\alpha_{n}^{\prime}\right)^{2}, \quad n=0,1,2, \ldots,
$$

the scheme (2.13) becomes

$$
\begin{aligned}
\phi_{0} & =q(x), \\
\phi_{n+1} & =q(x)+\left[\phi_{n}, x\right], \quad n=0,1,2, \ldots,
\end{aligned}
$$

where we have introduced the symbol

$$
[\phi, x] \equiv-\frac{1}{4} \frac{\phi^{\prime \prime}}{\phi}+\frac{5}{16}\left(\frac{\phi^{\prime}}{\phi}\right)^{2} \text {. }
$$


Then $\phi_{n}(x)$ will approximate $\phi(x)$, the solution to

$$
\phi=q(x)+[\phi, x],
$$

into which equation (2.9) is transformed by setting

$$
\phi \equiv\left(\alpha^{\prime}\right)^{2} \text {. }
$$

This procedure has the advantage of avoiding handling square roots, which causes complications when working in the complex plane. Moreover, using $\phi_{n}$ instead of $\alpha_{n}$ is also convenient in view of symbolic manipulations; cf. $\S 4$. In fact, (2.15) yields $\phi_{n}$ as a rational function of $q$ and its derivatives up to order $2 n$.

Note that $\{\alpha, x\}=-2[\phi, x]$ and that (2.17) is a second-order differential equation for $\phi$, while recursion (2.15) is no longer a differential equation for $\phi_{n+1}$. Only a single quadrature on $\phi_{n}^{1 / 2}(x)$ has to be accomplished at the end, for the value of $n$ related to a given accuracy.

Starting from the asymptotic assumptions (2.2)-(2.3) on the carrier $q(x)$, we now obtain the asymptotic behavior of the auxiliary function $\phi(x)$ which will be necessary for estimating the term $[\phi, x]$.

Since in proving the convergence of $\phi_{n}$ to $\phi$, estimates are needed on the derivatives of $\phi_{n}-\phi$ (cf. [ $\left.\phi_{n}, x\right]$ in (2.15)), we "complexify" all quantities involved, extending them from the real half line into a sector $S$. We start with a complex Liouville-Green basis in $S$. Following Olver [10], we split the carrier $q(z)$ into two parts, $f(z)$ and $g(z), q(z)=f(z)+g(z)$, where

$$
f(z)=a, \quad g(z)=Q(z)=O\left(z^{-p}\right) .
$$

Theorem 11.1 in [10, Chapter 6] yields the basis

$$
\begin{aligned}
& U(z)=f^{-1 / 4}(z) e^{i \xi(z)}\left[1+\varepsilon_{1}(z)\right], \\
& V(z)=f^{-1 / 4}(z) e^{-i \xi(z)}\left[1+\varepsilon_{2}(z)\right],
\end{aligned}
$$

where

$$
\xi(z)=\int^{z} f^{1 / 2}(t) d t
$$

and

$$
\begin{aligned}
& \left|\varepsilon_{j}(z)\right| \leq \exp \left\{V_{z, \infty}^{l_{j}}(F)\right\}-1, \\
& \left|\varepsilon_{j}^{\prime}(z)\right| \leq\left|f^{1 / 2}(z)\right|\left(\exp \left\{V_{z, \infty}^{l_{j}}(F)\right\}-1\right), \quad j=1,2 ;
\end{aligned}
$$

here $V_{z, \infty}^{l_{j}}(F)$ denotes the variation of $F$,

$$
F \equiv F(z) \equiv \int^{z}\left(f^{-1 / 4} D^{2} f^{-1 / 4}+g f^{-1 / 2}\right) d t,
$$

along the path $l_{j}, j=1,2$. The path $l_{j}$ connects $z$ to $\infty$ remaining inside $S_{\rho, \gamma}$, and has the property of being " $\xi$-progressive", i.e., $\operatorname{Im}[\xi(z)]$ is nonincreasing along $l_{1}$ and nondecreasing along $l_{2}$ (cf. [10, p. 222]). The splitting 
of $q$ must satisfy only the requirement that the variation $V_{z, \infty}^{l_{j}}(F)$ be finite for $j=1,2$ and all $z$ in $S_{\rho, \gamma}$. The choice (2.19) is acceptable.

Above, we chose for the square root the branch that is continuous and real positive for real positive argument; moreover, we stipulate that $\left(f^{-1 / 4}\right)^{2}=$ $f^{-1 / 2}$; cf. [10, p. 222]. Therefore, all quantities above, $f^{-1 / 2}(z), \xi(z), \varepsilon_{j}(z)$, $F(z), U(z), V(z)$, are holomorphic in the simply connected region $S_{\rho, \gamma}$. We get

$$
\xi(z)=a^{1 / 2} z
$$

where the constant of integration in (2.21) has been chosen equal to zero, with no loss of generality.

The variation of $F(z)$ introduced above is defined by

$$
V_{z, \infty}^{l_{j}}(F) \equiv \int_{t_{1}}^{t_{2}}\left|F^{\prime}(z(t))\right|\left|z^{\prime}(t)\right| d t,
$$

where the path $l_{j}$ is parametrically defined by $z=z(t), t_{1}<t<t_{2}$; cf. [10, Chapter 1, p. 29]. It is easy to check that, because of (2.24), if $\operatorname{Im} z \geq 0$, the straight half-line through the origin and $z \in S_{\rho, \gamma}$, joining $z$ to $\infty$, can be taken as the path $l_{1}$. As for $l_{2}$, we can take the circular arc with radius $|z|$ joining $z$ to the point $(|z|, 0)$ and then the real half line $x \geq|z|$ (see Figure 1). If $\operatorname{Im} z<0$, we interchange the roles of $l_{1}$ and $l_{2}$.

Therefore, we have

$$
\begin{gathered}
F(z)=a^{-1 / 2} \int^{z} Q(t) d t, \\
V_{z, \infty}^{l_{j}}(F) \leq \kappa(p) a^{-1 / 2}|z|^{1-p}, \quad j=1,2,
\end{gathered}
$$

where

$$
\kappa(p)=\left(\gamma+\frac{1}{p-1}\right) K
$$

( $\gamma$ could be omitted in (2.28) when estimating $V_{z, \infty}^{l_{1}}$ ). An estimate for $\varepsilon_{j}$ then follows:

$$
\begin{aligned}
\left|\varepsilon_{j}(z)\right| & \leq V_{z, \infty}^{l_{j}} \exp \left\{V_{z, \infty}^{l_{j}}\right\} \\
& <\kappa(p) a^{-1 / 2} \exp \left\{\kappa(p) a^{-1 / 2} \rho^{1-p}\right\}|z|^{1-p}, \quad j=1,2,
\end{aligned}
$$

since $|z|>\rho$ for any $z \in S_{\rho, \gamma}$. Note the double asymptotic nature in $\varepsilon_{j}(z)=$ $O\left(a^{-1 / 2} z^{1-p}\right)$ with respect to both the variable $z$, as $z \rightarrow \infty$ in $S_{\rho, \gamma}$, and the parameter $a$, as $a \rightarrow+\infty$. This fact is related to the well-known double asymptotic nature of the Liouville-Green approximations [10, Chapter 6].

At this point, we construct the real Liouville-Green basis, i.e., a linear combination of $U(z)$ and $V(z)$ which is real on the real axis. Recalling that $V(x)=\bar{U}(x)$ (cf. [10, Chapter 6]), we obtain the real basis

$$
u(x)=\operatorname{Re} U(x), \quad v(x)=\operatorname{Im} U(x) .
$$




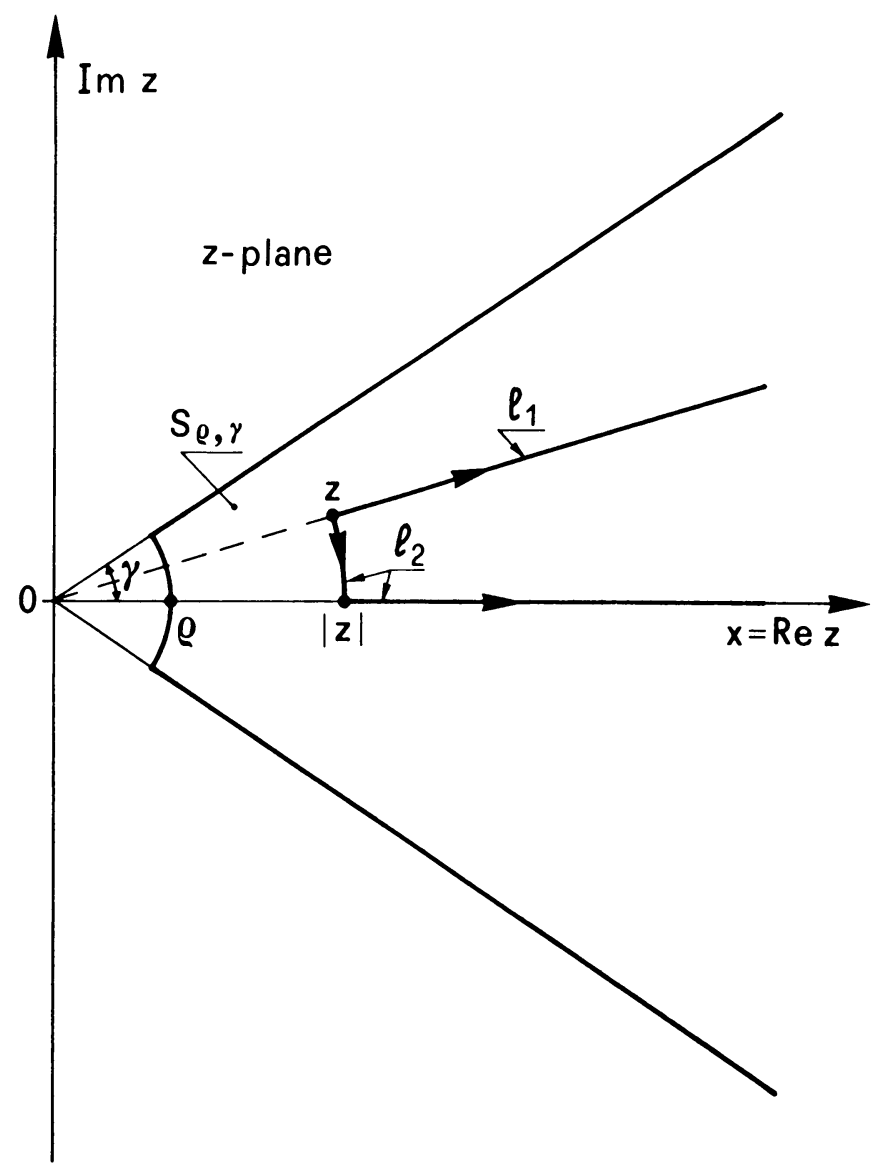

FIGURE 1

" $\xi$-progressive" paths in the $z$-plane for the case $\operatorname{Im} z>0$

It is clear now that $u(x), v(x)$ can be holomorphically continued (in a unique way) into $S_{\rho, \gamma}$ by the relations

$$
u(z) \equiv \frac{U(z)+V(z)}{2}, \quad v(z) \equiv \frac{U(z)-V(z)}{2 i} .
$$

Correspondingly, the auxiliary function $\phi(x)$ can be extended by

$$
\phi(z) \equiv \frac{W^{2}}{\left[u^{2}(z)+v^{2}(z)\right]^{2}} .
$$

This extension is valid, however, only in a subset of $S_{\rho, \gamma}$, where $u^{2}(z)+$ $v^{2}(z) \neq 0$.

This is, in fact, true in $S_{\rho, \gamma} \cap \mathbf{R}=\{x: x>\rho\}$, and in $S_{\rho, \gamma}$ as well, provided that

$$
u^{2}(z)+v^{2}(z)=U(z) V(z)=a^{-1 / 2}\left[1+\varepsilon_{1}(z)\right]\left[1+\varepsilon_{2}(z)\right] \neq 0,
$$

i.e., for $\left|\varepsilon_{j}(z)\right|<1, j=1,2$. This entails a possible restriction of $S_{\rho, \gamma}$ to an annular sector with a larger value of $\rho$ (but with unchanged angle). Below we 
shall use the same symbol for this modified sector. Equation (2.17) itself can be considered as a differential equation in $S_{\rho, \gamma}$.

We now obtain a preliminary asymptotic approximation for $\phi(z)$ as $z \rightarrow \infty$ in $S_{\rho, \gamma}$. Evaluating the (constant) Wronskian

$$
W[u(z), v(z)]=1
$$

through (2.20), we get from (2.32), (2.33), (2.29)

$$
\phi(z) \equiv \frac{a}{\left[1+\varepsilon_{1}(z)\right]^{2}\left[1+\varepsilon_{2}(z)\right]^{2}}=a\left[1+O\left(a^{-1 / 2} z^{1-p}\right)\right] .
$$

We can go further and improve this asymptotic approximation as follows. Using the asymptotic differentiation theorem [10, Chapter 1], we obtain from (2.35)

$$
\phi^{\prime}(z)=O\left(a^{1 / 2} z^{-p}\right), \quad \phi^{\prime \prime}=O\left(a^{1 / 2} z^{-p-1}\right), \quad z \in S_{\rho^{\prime}, \gamma^{\prime}},
$$

where $0<\gamma^{\prime} \equiv \gamma-\delta$ and $\rho^{\prime}=\rho /(1-\sin \delta), \delta$ being an arbitrary but fixed number with $0<\delta<\pi / 2$. The $O$-symbols in (2.36) depend on $\delta$.

Using this result in (2.16) we obtain

$$
\begin{aligned}
{[\phi, z] } & =-\frac{1}{4} \frac{O\left(a^{1 / 2} z^{-1-p}\right)}{a+O\left(a^{1 / 2} z^{1-p}\right)}+\frac{5}{16}\left[\frac{O\left(a^{1 / 2} z^{-p}\right)}{a+O\left(a^{1 / 2} z^{1-p}\right)}\right]^{2} \\
& =O\left(a^{-1 / 2} z^{-1-p}\right), \quad z \in S_{\rho^{\prime}, \gamma^{\prime}}
\end{aligned}
$$

and thus, from (2.17) (considered in $S_{\rho^{\prime}, \gamma^{\prime}}$ ),

$$
\phi(z)=q(z)+O\left(a^{-1 / 2} z^{-1-p}\right), \quad z \in S_{\rho^{\prime}, \gamma^{\prime}} .
$$

Remark 2.1. Note that a larger value of $a, a>0$, yields better estimates in (2.38). In particular, for $a \geq 1$ the estimates can be given uniformly in $a$.

We observe, in closing, that we had to pass through the complex LiouvilleGreen basis, as the phase functions were earlier defined only on the real line in Borůvka's theory.

\section{THE ITERATIVE SCHEME}

The numerical scheme consists of solving iteratively equations (2.15) for $\phi_{n}$, i.e.,

$$
\begin{aligned}
\phi_{0}(z) & =q(z), \\
\phi_{n+1}(z) & =q(z)+\left[\phi_{n}, z\right], \quad n=0,1,2, \ldots .
\end{aligned}
$$

Below, we shall prove the convergence of this scheme (in a suitable sense) to the solution of (2.17) corresponding to the Liouville-Green basis. For this purpose we need a basic lemma. Hereafter, for convenience, we change the independent variable $z$ by setting $z=\beta t$, with $\beta>0$ to be specified later.

Such a transformation takes equation (1.1) into

$$
\tilde{y}^{\prime \prime}+\tilde{q}(t) \tilde{y}=0,
$$


for $\tilde{y}(t)=y(\beta t)$, where

$$
\tilde{q}(t) \equiv \beta^{2} q(\beta t)=\beta^{2}\left[a+O\left(\beta^{-p} t^{-p}\right)\right]
$$

Below, we apply algorithm (3.1) to (3.2)-(3.3), thus obtaining

$$
\begin{aligned}
\tilde{\phi}_{0}(t) & =\tilde{q}(t), \\
\tilde{\phi}_{n+1}(t) & =\tilde{q}(t)+\left[\tilde{\phi}_{n}, t\right], \quad n=0,1,2, \ldots .
\end{aligned}
$$

These quantities approximate (in the sense defined in Theorem 3.2) the function

$$
\tilde{\phi}(t)=\frac{(\widetilde{W})^{2}}{\left[u^{2}(\beta t)+v^{2}(\beta t)\right]^{2}}
$$

a solution of

$$
\tilde{\phi}=\tilde{q}(t)+[\tilde{\phi}, t]
$$

It is easily shown that $\tilde{\phi}(t)=\beta^{2} \phi(\beta t)$ and $\tilde{\phi}_{n}(t)=\beta^{2} \phi_{n}(\beta t)$. Our objective is to approximate $\phi$, but we find it more convenient to first consider $\tilde{\phi}$ and then to go back to $\phi$.

Lemma 3.1. Let

$$
G\left(z_{1}, z_{2}, z_{3}\right) \equiv \tilde{q}-\frac{1}{4} \frac{z_{3}}{z_{1}}+\frac{5}{16}\left(\frac{z_{2}}{z_{1}}\right)^{2}
$$

be a complex-valued function of the three complex variables $z_{1}, z_{2}, z_{3}$, defined on the closed polydisc $\bar{P}=\bar{D}_{1} \times \bar{D}_{2} \times \bar{D}_{3}$, where $D_{1}=B\left(c, \varepsilon_{1}\right), D_{2}=B\left(0, \varepsilon_{2}\right)$, and $D_{3}=B\left(0, \varepsilon_{3}\right)$, with $B\left(z_{0}, r\right) \subset \mathbf{C}$ denoting the open ball with center in $z_{0}$ and radius $r$ and $0<\varepsilon_{1}<c$ ( $c$ a positive constant), $\varepsilon_{2}>0, \varepsilon_{3}>0 ; \tilde{q} \in \mathbf{C}$ is a constant. Then $G$ is uniformly Lipschitz continuous in $\bar{P}$,

$$
|G(\mathbf{z})-G(\boldsymbol{\eta})| \leq \chi\|\mathbf{z}-\boldsymbol{\eta}\|, \quad \forall \mathbf{z}, \boldsymbol{\eta} \in \bar{P},
$$

where $\mathbf{z} \equiv\left(z_{1}, z_{2}, z_{3}\right), \boldsymbol{\eta} \equiv\left(\eta_{1}, \eta_{2}, \eta_{3}\right)$, and

$$
\chi=\frac{\sqrt{3}}{4} \max \left\{\frac{\varepsilon_{3}}{\left(c-\varepsilon_{1}\right)^{2}}+\frac{5 \varepsilon_{2}^{2}}{2\left(c-\varepsilon_{1}\right)^{3}}, \frac{5 \varepsilon_{2}}{2\left(c-\varepsilon_{1}\right)^{2}}, \frac{1}{c-\varepsilon_{1}}\right\} .
$$

Proof. The function $G$ is holomorphic in $\bar{P}$ (being separately holomorphic in each of the $z_{i}$ 's, by Hartog's theorem). We obtain

$$
\frac{\partial G}{\partial z_{1}}=\frac{z_{3}}{2 z_{1}^{2}}-\frac{5}{8} \frac{z_{2}^{2}}{z_{1}^{3}}, \quad \frac{\partial G}{\partial z_{2}}=\frac{5}{8} \frac{z_{2}}{z_{1}^{2}}, \quad \frac{\partial G}{\partial z_{3}}=-\frac{1}{4 z_{1}},
$$

and then, by the mean-value theorem,

$$
|G(\mathbf{z})-G(\boldsymbol{\eta})| \leq \sup _{\boldsymbol{\tau} \in[\mathbf{z}, \boldsymbol{\eta}]}\{\|\operatorname{grad} G(\boldsymbol{\tau})\|\} \cdot\|\mathbf{z}-\boldsymbol{\eta}\| ;
$$

cf. [7]. In fact, this property holds for $C^{1}$-mappings $G:\left(\mathbf{U} \subset \mathbf{B}_{1}\right) \rightarrow \mathbf{B}_{2}$, where $\mathbf{U}$ is a convex subset of $\mathbf{B}_{1}$, and $\mathbf{B}_{1}$ and $\mathbf{B}_{2}$ are Banach spaces. In (3.11), 
$[\mathbf{z}, \boldsymbol{\eta}]$ denotes the segment in $\mathbf{C}^{3}$ joining $\mathbf{z}$ and $\boldsymbol{\eta}$, which is obviously a subset of $\bar{P}$ for any $\mathbf{z}, \eta \in \bar{P}$, since $\bar{P}$ is a convex set in $\mathbf{C}^{3}$. The norms on the right-hand side of (3.11) represent the Euclidean norms in $\mathbf{C}^{3}$. Using (3.11) we get (3.8), (3.9), since

$$
\|\operatorname{grad} G(\boldsymbol{\tau})\| \leq \sqrt{3} \max _{1 \leq i \leq 3}\left|\frac{\partial G(\tau)}{\partial z_{i}}\right|,
$$

and

$$
\begin{aligned}
\left|\frac{\partial G}{\partial z_{1}}\right| & \leq \frac{1}{4} \frac{\varepsilon_{3}}{\left(c-\varepsilon_{1}\right)^{2}}+\frac{5}{8} \frac{\varepsilon_{2}^{2}}{\left(c-\varepsilon_{1}\right)^{3}}, \\
\left|\frac{\partial G}{\partial z_{2}}\right| & \leq \frac{5}{8} \frac{\varepsilon_{2}}{\left(c-\varepsilon_{1}\right)^{2}}, \quad\left|\frac{\partial G}{\partial z_{3}}\right| \leq \frac{1}{4} \frac{1}{\left(c-\varepsilon_{1}\right)},
\end{aligned}
$$

uniformly in $\bar{P}$. The lemma is thus proved.

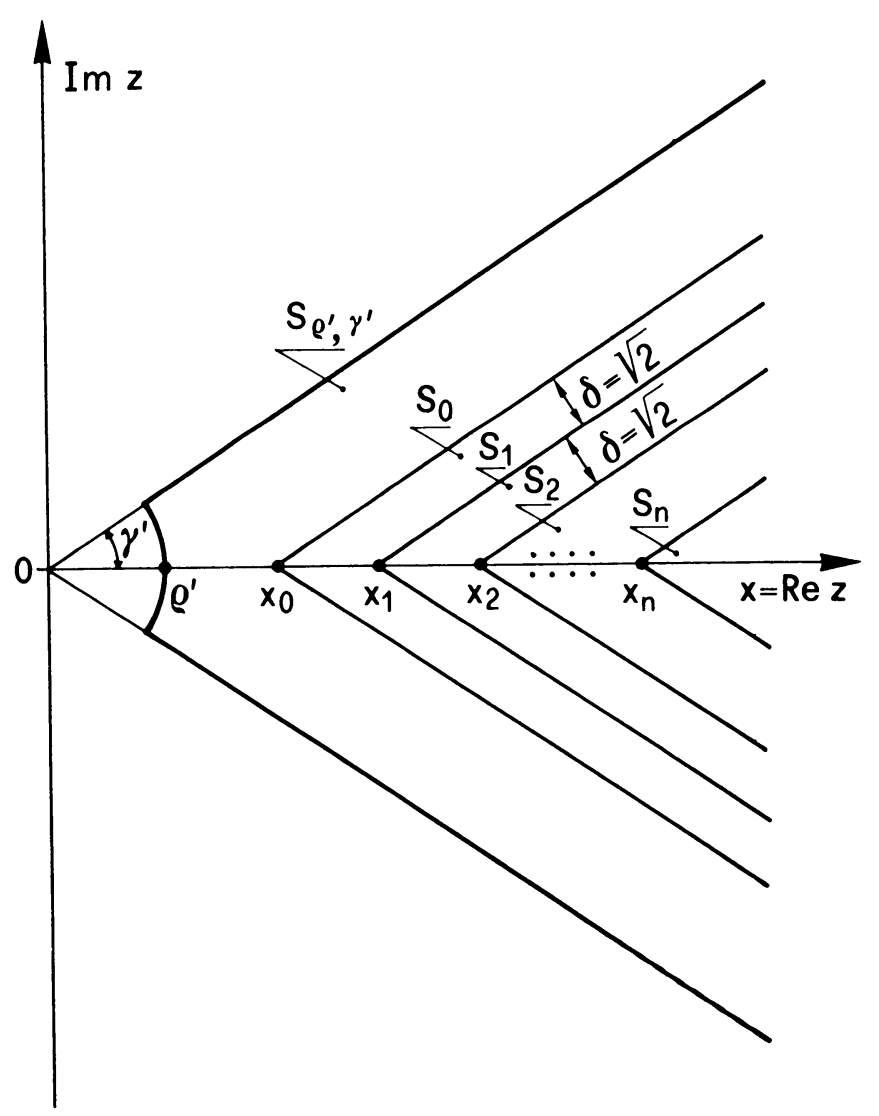

Figure 2

The decreasing sequence of sectors $S_{n}$ in the z-plane, used in Theorem 3.2 
Consider the iterative scheme (3.4) and recall that $S_{\rho^{\prime}, \gamma^{\prime}}$ is the (annular) sector in which the estimate $(2.38)$ holds. We denote by $S\left(0, \gamma^{\prime}\right)$ the sector having its vertex at the origin and semiangle $\gamma^{\prime}$, and we consider a sequence of sectors

$$
S_{n} \equiv x_{n}+S\left(0, \gamma^{\prime}\right) \text {, }
$$

where $x_{n}$, their vertices, is an increasing sequence of positive numbers (see Figure 2). At this point we state our main result as a theorem.

Theorem 3.2. Suppose that equation (1.1) is given with the carrier $q(z)$ satisfying all hypotheses stated in $\S 2$. Let $\beta$ be any fixed real number with $\beta>a^{-1 / 2}$. Then there exists $x_{0} \in S_{\rho^{\prime}, \gamma^{\prime}} \cap \mathbf{R}$, which depends on all parameters entering the problem (but not on $\beta$ ), such that, if $S_{n}$ is the sector defined in (3.14) with

$$
x_{n}=x_{0}+\frac{n \beta \sqrt{2}}{\sin \gamma^{\prime}},
$$

then the function $\phi_{n}(z)$ given by the scheme (3.1) is holomorphic in $S_{n}$ and "converges" to the function $\phi(z)$ related to the Liouville-Green basis by (2.32).

The convergence is in the sense that

$$
\left|\phi_{n}(z)-\phi(z)\right|=a^{-1 / 2}\left(\beta a^{1 / 2}\right)^{-2 n} O\left((|z|-n \beta \sqrt{2})^{-1-p}\right),
$$

for $z \in S_{n}$ and $n=0,1,2, \ldots$.

Proof. The proof proceeds inductively on $n \in \mathbf{N}$. We first change $z$ into $t$, $z=\beta t$ in our problem.

The transformation $z=\beta t$ takes equation (1.1) into (3.2) and its carrier $q(z)$ into $\tilde{q}(z)$ given by (3.3). Correspondingly, the Liouville-Green basis of (1.1), $(u(z), v(z))$, is transformed into $(\tilde{u}(t), \tilde{v}(t))$. Let $\tilde{\phi}(t)$ be the function defined in (3.5), i.e., the quantity corresponding to $\phi$ for equation (3.2), (3.3). Note that this is not the transform of $\phi$ under $z=\beta t$. The initial sector $S_{\rho^{\prime}, \gamma^{\prime}}$ of the complex $z$-plane is transformed into the sector $\widetilde{S}_{r^{\prime}, \gamma^{\prime}}$, where $r^{\prime}=\rho^{\prime} / \beta$, of the complex $t$-plane.

As $\tilde{\phi}(t)=\beta^{2} \phi(\beta t)$ and $\tilde{\phi}_{n}(t)=\beta^{2} \phi_{n}(\beta t)$, the estimate (2.38) becomes

$$
\tilde{\phi}(t)=\tilde{q}(t)+\beta^{1-p} a^{-1 / 2} O\left(t^{-1-p}\right), \quad t \in \tilde{S}_{r^{\prime}, \gamma^{\prime}} .
$$

As for the derivatives, we get from (2.36)

$$
\tilde{\phi}^{\prime}(t)=a^{1 / 2} \beta^{3-p} O\left(t^{-p}\right), \quad \tilde{\phi}^{\prime \prime}(t)=a^{1 / 2} \beta^{3-p} O\left(t^{-p-1}\right), \quad t \in \tilde{S}_{r^{\prime}, \gamma^{\prime}} .
$$

We are now ready for the inductive proof. For $n=0$, we have from (3.17),

$$
\left|\tilde{\phi}(t)-\tilde{\phi}_{0}(t)\right|=|\tilde{\phi}(t)-\tilde{q}(t)|=\beta^{1-p} a^{-1 / 2} O\left(t^{-1-p}\right), \quad t \in \tilde{S}_{r^{\prime}, \gamma^{\prime}} .
$$

Suppose that the estimate

$$
\left|\tilde{\phi}_{n}(t)-\tilde{\phi}(t)\right|=\beta^{1-p} a^{-1 / 2}\left(\beta a^{1 / 2}\right)^{-2 n} O\left((|t|-n \sqrt{2})^{-1-p}\right)
$$


holds in the sector $\widetilde{S}_{n}$ with vertex in $t_{n} \equiv x_{0} / \beta+n \sqrt{2} / \sin \gamma^{\prime}$, for some $x_{0} \geq \rho^{\prime}$ (to be determined below); cf. (3.15). Then, as in the proof of Lemma 3.1,

$$
\begin{aligned}
\left|\tilde{\phi}(t)-\tilde{\phi}_{n+1}(t)\right| & =\left|G\left(\tilde{\phi}, \tilde{\phi}^{\prime}, \tilde{\phi}^{\prime \prime}\right)-G\left(\tilde{\phi}_{n}, \tilde{\phi}_{n}^{\prime}, \tilde{\phi}_{n}^{\prime \prime}\right)\right| \\
& \leq \sup _{\boldsymbol{\tau} \in\left[\mathbf{w}, \mathbf{w}_{n}\right]}\{\|\operatorname{grad} G(\boldsymbol{\tau})\|\}\left\|\mathbf{w}-\mathbf{w}_{n}\right\|,
\end{aligned}
$$

where $\mathbf{w} \equiv\left(\tilde{\phi}, \tilde{\phi}^{\prime}, \tilde{\phi}^{\prime \prime}\right) \in \mathbf{C}^{3}$ and $\mathbf{w}_{n} \equiv\left(\tilde{\phi}_{n}, \tilde{\phi}_{n}^{\prime}, \tilde{\phi}_{n}^{\prime \prime}\right) \in \mathbf{C}^{3}$ depend on $t$. We still have, however, to identify a polydisc in which Lemma 3.1 holds. In estimating the norm $\left\|\mathbf{w}-\mathbf{w}_{n}\right\|$, we use (3.20) in the Cauchy formula to get

$$
\left|\tilde{\phi}^{(j)}(t)-\tilde{\phi}_{n}^{(j)}(t)\right| \leq \frac{j !}{(\sqrt{2})^{j}} \sup _{\zeta \in C}\left|\tilde{\phi}(\zeta)-\tilde{\phi}_{n}(\zeta)\right|, \quad t \in \widetilde{S}_{n+1},
$$

where $C$ denotes the circle with center in $t$ and radius $\sqrt{2}$. The sup in (3.22) is estimated by (3.20) using the structure of the sequence of sectors $\widetilde{S}_{n}$. Thus,

$$
\begin{aligned}
\left|\tilde{\phi}^{(j)}(t)-\tilde{\phi}_{n}^{(j)}(t)\right| \leq & \frac{j !}{(\sqrt{2})^{j}} \beta^{1-p} a^{-1 / 2}\left(\beta a^{1 / 2}\right)^{-2 n} \\
& \times O\left([|t|-(n+1) \sqrt{2}]^{-1-p}\right), \quad t \in \widetilde{S}_{n+1} .
\end{aligned}
$$

It is clear, finally, that

$$
\begin{aligned}
\left\|\mathbf{w}-\mathbf{w}_{n}\right\| \leq & \sqrt{3} \max \left\{\left|\tilde{\phi}-\tilde{\phi}_{n}\right|,\left|\tilde{\phi}^{\prime}-\tilde{\phi}_{n}^{\prime}\right|,\left|\tilde{\phi}^{\prime \prime}-\tilde{\phi}_{n}^{\prime \prime}\right|\right\} \\
= & \sqrt{3} \beta^{1-p} a^{-1 / 2}\left(\beta a^{1 / 2}\right)^{-2 n} \\
& \cdot O\left[(|t|-(n+1) \sqrt{2})^{-1-p}\right], \quad t \in \tilde{S}_{n+1} .
\end{aligned}
$$

In order to estimate $\|\operatorname{grad} G\|$ by Lemma 3.1, we have to estimate the radii of the component discs of the polydisc. We have

$$
\begin{aligned}
\left|\tilde{\phi}_{n}(t)-\beta^{2} a\right| & \leq\left|\tilde{\phi}(t)-\beta^{2} a\right|+\left|\tilde{\phi}_{n}(t)-\tilde{\phi}(t)\right| \\
& \leq \beta^{2-p} O\left(t^{-p}\right)+\beta^{1-p} a^{-1 / 2}\left(\beta a^{1 / 2}\right)^{-2 n} O\left((|t|-n \sqrt{2})^{-1-p}\right),
\end{aligned}
$$

and then, as $\beta a^{1 / 2}>1$, the estimate

$$
\left|\tilde{\phi}_{n}(t)-\beta^{2} a\right| \leq \beta^{2-p} O\left(\left(\frac{x_{0}}{\beta}\right)^{-p}\right)+\beta^{1-p} a^{-1 / 2} O\left(\left(\frac{x_{0}}{\beta}\right)^{-p-1}\right) \equiv \varepsilon_{3},
$$

say, uniformly in $n$ and $t \in \widetilde{S}_{n+1}$. Similarly, noticing that

$$
\begin{aligned}
\left|\tilde{\phi}^{\prime}(t)\right| & \leq a^{1 / 2} \beta^{3-p} O\left(\left(\frac{z}{\beta}\right)^{-p}\right)=\beta^{2} a^{1 / 2}\left(\frac{\beta}{|z|}\right) O\left(z^{1-p}\right) \\
& <\beta^{2} a^{1 / 2} O\left(z^{1-p}\right)<\beta^{2} a^{1 / 2} O\left(x_{0}^{1-p}\right), \quad \text { for } t \in \widetilde{S}_{n+1},
\end{aligned}
$$

as $|z| \geq x_{0}+(n+1) \beta \sqrt{2} / \sin \gamma^{\prime}$, and thus $|z|>x_{0}$ as well as $|z|>\sqrt{2} \beta$, and

$$
\begin{aligned}
\left|\tilde{\phi}^{\prime \prime}(t)\right| & \leq a^{1 / 2} \beta^{3-p} O\left[\left(\frac{z}{\beta}\right)^{-p-1}\right]=\beta^{2} a^{1 / 2}\left(\frac{\beta}{|z|}\right)^{2} O\left(z^{-p+1}\right) \\
& <\beta^{2} a^{1 / 2} O\left(x_{0}^{-p+1}\right), \quad \text { for } t \in \widetilde{S}_{n+1}
\end{aligned}
$$


we obtain

$$
\begin{aligned}
\left|\tilde{\phi}_{n}^{\prime}(t)\right| & \leq\left|\tilde{\phi}^{\prime}(t)\right|+\left|\tilde{\phi}^{\prime}(t)-\tilde{\phi}_{n}^{\prime}(t)\right| \\
& \leq \beta^{2} a^{1 / 2} O\left(x_{0}^{1-p}\right)+\beta^{1-p} a^{-1 / 2} O\left[\left(\frac{x_{0}}{\beta}\right)^{-p-1}\right] \\
& =\beta^{2}\left[a^{1 / 2} O\left(x_{0}^{1-p}\right)+a^{-1 / 2} O\left(x_{0}^{-p-1}\right)\right] \equiv \varepsilon_{2}, \\
\left|\tilde{\phi}_{n}^{\prime \prime}(t)\right| & \leq\left|\tilde{\phi}^{\prime \prime}(t)\right|+\left|\tilde{\phi}^{\prime \prime}(t)-\tilde{\phi}_{n}^{\prime \prime}(t)\right| \\
& \leq \beta^{2} a^{1 / 2} O\left(x_{0}^{-p+1}\right)+\beta^{1-p} a^{-1 / 2} O\left[\left(\frac{x_{0}}{\beta}\right)^{-p-1}\right] \\
& =\beta^{2}\left[a^{1 / 2} O\left(x_{0}^{-p+1}\right)+a^{-1 / 2} O\left(x_{0}^{-p-1}\right)\right] \equiv \varepsilon_{1} .
\end{aligned}
$$

Writing

$$
\varepsilon_{i}=f_{i}\left(x_{0}, a\right) \beta^{2} a, \quad i=1,2,3,
$$

$f_{i}$ being defined by $\left(3.24^{\prime}\right),(3.26)$, we first claim that $1 /\left(c-\varepsilon_{3}\right) \leq \frac{4}{3}\left(1 / \beta^{2} a\right)$, with $c \equiv \beta^{2} a$. This entails the estimate

$$
0<\varepsilon_{3} \leq \frac{\beta^{2} a}{4}
$$

which can be satisfied by choosing $x_{0}$ sufficiently large, say $x_{0} \geq x_{0}^{\prime}$, since $f_{i}\left(x_{0}, a\right)$ is infinitesimal as $x_{0} \rightarrow+\infty$. Now we observe that $\left(3.24^{\prime}\right)$ ensures that $\tilde{\phi}_{n}$ dees not vanish in $\widetilde{S}_{n+1}$, and therefore $\tilde{\phi}_{n+1}$ is holomorphic in $\widetilde{S}_{n+1}$. We then claim $\chi \leq(\sqrt{3 / 4}) /\left(c-\varepsilon_{3}\right)\left(c \equiv \beta^{2} a\right)$, i.e., we can choose $\varepsilon_{1}$ and $\varepsilon_{2}$ in such a way that the other two components of $\operatorname{grad} G$ are estimated by $\frac{4}{3}\left(1 / \beta^{2} a\right)$. This can be done again, because $f_{i}$ is infinitesimal, for $x_{0}$ sufficiently large, say $x_{0} \geq x_{0}^{\prime \prime}$. We conclude that

$$
\chi \leq \frac{\sqrt{3}}{3} \frac{1}{\beta^{2} a}
$$

and hence, by Lemma 3.1, using (3.21) and (3.23), we get

$$
\begin{aligned}
\mid \tilde{\phi}(t) & -\tilde{\phi}_{n+1}(t) \mid \\
& \leq \frac{\sqrt{3}}{3} \frac{1}{\beta^{2} a} \sqrt{3} \beta^{1-p} a^{-1 / 2}\left(\beta a^{1 / 2}\right)^{-2 n} O\left[(|t|-(n+1) \sqrt{2})^{-1-p}\right] \\
& =\beta^{1-p} a^{-1 / 2}\left(\beta^{2} a\right)^{-(n+1)} O\left[(|t|-(n+1) \sqrt{2})^{-1-p}\right], \quad t \in \widetilde{S}_{n+1},
\end{aligned}
$$

provided that $x_{0} \geq \max \left\{\rho^{\prime}, x_{0}^{\prime}, x_{0}^{\prime \prime}\right\}$. This value of $x_{0}$ depends, in general, on all parameters entering the problem (except $\beta$ ). Comparing (3.30) with (3.20) and going back to the variable $z$, it is clear that the inductive proof is complete.

Some observations are now in order. In the proof above, the condition $\beta^{2} a>1$ was needed. The transformation $z=\beta t$ with $\beta>a^{-1 / 2}$ is therefore 
instrumental in reducing equation (1.1) to an equation to which the algorithm (3.1) can be applied when $a<1$. Moreover, it is clear from (3.16) that the larger $\beta^{2} a$ the faster the "convergence" of the algorithm. However, larger values of $\beta$ reduce the $\operatorname{Re}(t)$-interval for a given $x$-interval $(x=\operatorname{Re}(z))$ in which we want to evaluate zeros. Therefore, the zeros are not so well separated when we choose $\beta$ large.

Going back to the $z$-variable, the vertices of the sectors $S_{n}$ increase linearly with $\beta$ (and with $n$ ); cf. (3.15). This entails that the zeros lying outside $S_{n}$ cannot be approximated by using the estimate in (3.16) for $\phi$.

As a corollary of Theorem 3.2, we can relate the desired accuracy to the number of iterations needed to attain it.

Corollary 3.3. The number of iterations, $n(\varepsilon)$, needed to attain the accuracy $\varepsilon$ in the iterative scheme (3.1) is asymptotically given by

$$
n(\varepsilon) \sim \frac{|\log \varepsilon|}{\log \left(\beta^{2} a\right)}, \quad \varepsilon \rightarrow 0^{+},
$$

uniformly in $z, z \in S_{n(\varepsilon)}$.

Proof. From (3.16), the condition $\left|\phi_{n}(z)-\phi(z)\right| \leq \varepsilon$, with $|z|-n \beta \sqrt{2} \geq x_{0}$ for all $z$ in $S_{n}$, i.e.,

$$
a^{-1 / 2}\left(\beta^{2} a\right)^{-n} O\left(x_{0}^{-1-p}\right) \leq \varepsilon, \quad \forall z \in S_{n},
$$

is certainly satisfied when $n \geq(C-\log \varepsilon) / \log \left(\beta^{2} a\right)$ for some constant $C$, and thus choosing

$$
n=n(\varepsilon) \sim \frac{|\log \varepsilon|}{\log \left(\beta^{2} a\right)}, \quad \varepsilon \rightarrow 0^{+}, \quad z \in S_{n(\varepsilon)} .
$$

In the next section, we shall numerically compute zeros of solutions to equation (1.1) starting from the approximation $\phi_{n}$ of the function $\phi$. If we approximate $\phi$ within an accuracy $\varepsilon$, i.e., use $\phi_{n(\varepsilon)}$, we "lose" zeros in the sense that our procedure is asymptotic in nature, and only those zeros lying in $S_{n(\varepsilon)}$ can be approximated. In fact, we get from [5, Chapter XI, Corollary 5.3, p. 348] the asymptotic estimate

$$
N(\rho, x) \sim \frac{\sqrt{a}}{\pi} x \quad \text { as } x \rightarrow+\infty
$$

for the number of zeros in the interval $(\rho, x)$. Using (3.31) in

$$
x_{n(\varepsilon)} \equiv x_{0}+\frac{\beta \sqrt{2}}{\sin \gamma^{\prime}} n(\varepsilon) \sim \frac{\beta \sqrt{2}}{\sin \gamma^{\prime}} n(\varepsilon),
$$

and this in (3.32) with $x=x_{n(\varepsilon)}$, we obtain

$$
N\left(\rho, x_{n(\varepsilon)}\right) \sim A|\log \varepsilon| \text { as } \varepsilon \rightarrow 0^{+},
$$

where

$$
A \equiv \frac{\beta \sqrt{2 a}}{\pi \sin \gamma^{\prime} \log \left(\beta^{2} a\right)} .
$$


This shows the advantage of our approach compared to the mere asymptotic approximation $\left(\phi-\phi_{0} \equiv q\right)$. In fact, in order to obtain the same degree of accuracy, the latter requires $x \geq \bar{x}(\varepsilon) \equiv\left(B a^{-1 / 2}\right)^{1 /(1+p)} \varepsilon^{-1 /(1+p)}$, where $B$ is the constant in the $O$-symbol in (2.38). We emphasize, finally, that our approach is not only better because of logarithmic growth as opposed to power growth as $\varepsilon \rightarrow 0^{+}$, but also because we made coarse estimates in obtaining (3.31). Better results could have been obtained by taking into account the $z$-dependence in (3.16).

Remark 3.4. Theorem 3.2 also holds for the more general class of carriers

$$
\begin{aligned}
& q(z)=a+\frac{b}{z}+Q(z), \quad a>0, b \in \mathbf{R}, \\
& Q(z)=O\left(z^{-p}\right), \quad p>1 .
\end{aligned}
$$

This form allows us to include carriers having full asymptotic or convergent power series expansions in $z^{-1}$ like $\sum_{j=0}^{\infty} a_{j} z^{-j}$. We can write

$$
q(z)=\left(\sqrt{a}+\frac{b}{2 \sqrt{a}} \frac{1}{z}\right)^{2}+O\left(z^{-\mu}\right), \quad \mu=\min \{p, 2\},
$$

and split $q(z)$ as $q(z)=f(z)+g(z)$, with

$$
f(z) \equiv\left(\sqrt{a}+\frac{b}{2 \sqrt{a}} \frac{1}{z}\right)^{2}, \quad g(z) \equiv Q(z)-\frac{b^{2}}{4 a} \frac{1}{z^{2}} .
$$

Indeed, in this case it is easily shown that the $\xi$-progressive paths are unchanged with respect to the case $b=0$, the variation defined in (2.25) being correspondingly finite at the same time. We shall choose $|z|>|b| / 2 a$, which amounts to selecting the principal branch of $f^{1 / 2}(z)$. This entails, possibly, an increase of the radius $\rho$ in the annular sector, $S_{\rho, \gamma}$. Moreover, it turns out that $V_{z, \infty}^{l_{j}}=O\left(z^{-\lambda}\right)$, where $\lambda=\min \{p-1,1\}$, and then $\varepsilon_{j}=O\left(z^{-\lambda}\right)$, $\phi(z)=q(z)+O\left(z^{-\lambda-2}\right)$. We conclude that Theorem 3.2 holds with $\lambda+2$ replacing $p+1$. We observe, in closing, that the $O$-symbols in the estimate of $V_{z, \infty}^{l_{j}}$, and thus in the estimates of $\varepsilon_{j}$ and $\phi$, depend in general on $b$ (in addition to $a, \rho, \gamma, p, K$, as before).

\section{NUMERICAL EXAMPLES}

If $\alpha(x)$ denotes a Liouville-Green phase, then the relation (2.7) and the definition (2.18) ensure that the equation

$$
\alpha\left(x_{k}\right)-\alpha(x)=\int_{x}^{x_{k}} \phi^{1 / 2}(t) d t=\pi
$$

$x_{k}$ being any fixed zero, has the unique solution $x=x_{k-i}$ (relation (4.1) actually refers to the Liouville-Green basis $(v, u))$. In practice we solve, instead, equation

$$
\int_{x}^{\tilde{x}_{k}} \phi_{n}^{1 / 2}(t) d t-\pi=0
$$


In fact, we start from an approximation $\tilde{x}_{h}$ of a certain "large" zero, $x_{h}$, and compute successively the approximate values of the smaller zeros $x_{k}$, $k=h-1, h-2, \ldots$, which are denoted by $\tilde{x}_{k}$. The initial value $\tilde{x}_{h}$ must be supplied independently of our method. Moreover, throughout all of the procedure, the approximation $\phi_{n}$ of $\phi$ is used, the corresponding error being given by Theorem 3.2.

Therefore, in evaluating zeros of solutions to (1.1), we can identify two main steps. We have first to obtain an approximation $\phi_{n}$ of $\phi$ (which is accomplished by Theorem 3.2, see the algorithm (2.15)), and then to solve equation (4.2). As for the first step, note that $\phi_{n}$ turns out to be a rational function of $q, q^{\prime}, \ldots, q^{(2 n)}$ (cf. (2.15)), and one can take advantage of computer algebra techniques to get such a function explicitly. In fact, in the examples below, we used MACSYMA. Moreover, whenever $q$ is given in terms of elementary functions, $\phi_{n}$ is of the same type; in particular, if $q$ is a rational function, $\phi_{n}$ is also rational.

Following this procedure, the numerator and denominator degrees of the rational functions involved in general increase exponentially with $n$, thus yielding exponential computational complexity. The convergence of the algorithm is, however, very fast (cf. (3.16)), so that accurate results are obtained in very few iterations. On the other hand, if we merely use numerical differentiations in (2.15), the propagation of the local truncation error cannot be controlled after a few iterations.

In the examples below, the carrier $q$ is given in terms of elementary functions; we then proceed as follows, combining conveniently symbolic manipulations and numerical evaluations in order to face only polynomial computational complexity. It is easily seen that the evaluation of $\phi_{n}$ at a certain point requires knowing $q, q^{\prime}, \ldots, q^{(2 n)}$ at that point (this can be accomplished by MACSYMA). In fact, $\phi_{n}$ requires knowing $\phi_{n-1}, \phi_{n-1}^{\prime}, \phi_{n-1}^{\prime \prime}$, which, in turn, require $\phi_{n-2}, \phi_{n-2}^{\prime}, \ldots, \phi_{n-2}^{(4)}$, so that, at the end, we need $\phi_{1}, \phi_{1}^{\prime}, \ldots, \phi_{1}^{(2 n-2)}$, and finally $q, q^{\prime}, \ldots, q^{(2 n)}$. In computing $\phi_{j}$ and its derivatives (up to the order we need) we have to calculate derivatives of quotients involving $\phi_{j-1}$. This can be done via the simple algorithm used to compute ratios of formal series (cf. [10, p. 20]). The computational complexity involved in such a procedure is of order $O\left(n^{3}\right)$. Observe, finally, that using $\phi_{n}$ instead of $\alpha_{n}^{\prime}$ throughout the paper has the advantage of avoiding square roots at each iteration. Basing our method on $\alpha_{n}^{\prime}$ directly would prevent using the previous techniques.

As for the second step, we just solve equation (4.2) by the Newton-Raphson method. This requires only few numerical quadratures of $\phi_{n}^{1 / 2}(\cdot)$. Notice that square roots are evaluated only corresponding to the last value of the iteration number, $n$.

Example 1. As a first illustration, we computed the smallest 19 zeros of the Bessel function $J_{0}(x)$. Here, $\sqrt{x} J_{0}(x)$ is a solution of equation (1.1) with the carrier $q(x)=1-\left(\nu^{2}-1 / 4\right) / x^{2}$ with $\nu=0$, which belongs to the class 
described in $\S 2$. We started from the 20th zero of $J_{0}(x), j_{0,20}$, that is tabulated with 10 decimals in [1]. In Table 1, we list the computed values of the zeros $\tilde{x}_{k}$, along with the discrepancy between them and the tabulated values, for four values of the iteration number, $n=0,1,2,3$. In solving equation (4.2) for each zero by the Newton-Raphson method, two or three iterations were sufficient.

Example 2. In Table 2, we show results similar to the previous ones for the zeros of the Bessel function of second kind, $Y_{5}(x)$. Its 20th zero, $y_{5,20}$ is given in [9] with eight decimals.

Example 3. In order to exhibit other nontrivial cases, we constructed some examples starting from the explicit form of a phase function, $\alpha(x)$. Recall that any given phase function uniquely identifies the carrier of (1.1) by relation (2.9) as well as the associated basis, $\left(y_{1}, y_{2}\right), y_{1} \equiv\left|\alpha^{\prime}\right|^{-1 / 2} \sin \alpha, y_{2} \equiv\left|\alpha^{\prime}\right|^{-1 / 2} \cos \alpha$. Choosing $\alpha(x)=x+1 / x, x>0$, we get

$$
q(x)=\frac{x^{8}-4 x^{6}+3 x^{4}-4 x^{2}+1}{x^{8}-2 x^{6}+x^{4}} .
$$

This lies in the class described in $\S 2$.

In Table 3 we list the zeros of the solution $y_{1}(x)$, i.e., the roots of the equation

$$
x+1 / x=k \pi, \quad k=1,2, \ldots, 19 ;
$$

these can be computed directly as

$$
x_{k} \equiv \frac{k \pi+\left(k^{2} \pi^{2}-4\right)^{1 / 2}}{2}
$$

for the purpose of comparison. The starting point $x_{20}$ was computed from this formula (we neglected those zeros clustering near $x=0$ ). Table 3 is organized in the same way as Tables 1 and 2.

Example 4. We produce another example proceeding as in Example 3. Choosing $\alpha(x)=x+\log x / x^{2}, x>0$, we obtain again a carrier to which Theorem 3.2 can be applied. Here it was convenient to use MACSYMA in producing the derivatives $q^{(j)}$ of $q$, since $q$ is not rational. The zeros of $y_{1}(x)$, i.e., the roots of

$$
x+\log x / x^{2}=k \pi, \quad k=1,2, \ldots, 19,
$$

are computed by a Newton-Raphson procedure and compared with the results of our algorithm; see Table 4. In particular, the 20th zero from which we started was obtained in this way.

Remark 4.1. Inspection of the tables shows that, for any fixed $n$, better results are obtained for larger $k$ 's. This can be explained by the fact that the method is asymptotic in nature; cf. (3.16). Moreover, there is numerical evidence that by increasing $n$, the larger zeros are better approximated; this is in accordance with Theorem 3.2. The errors in computing the smallest zeros, on the other hand, are bigger and tend to stabilize or even to increase when $n$ increases. 


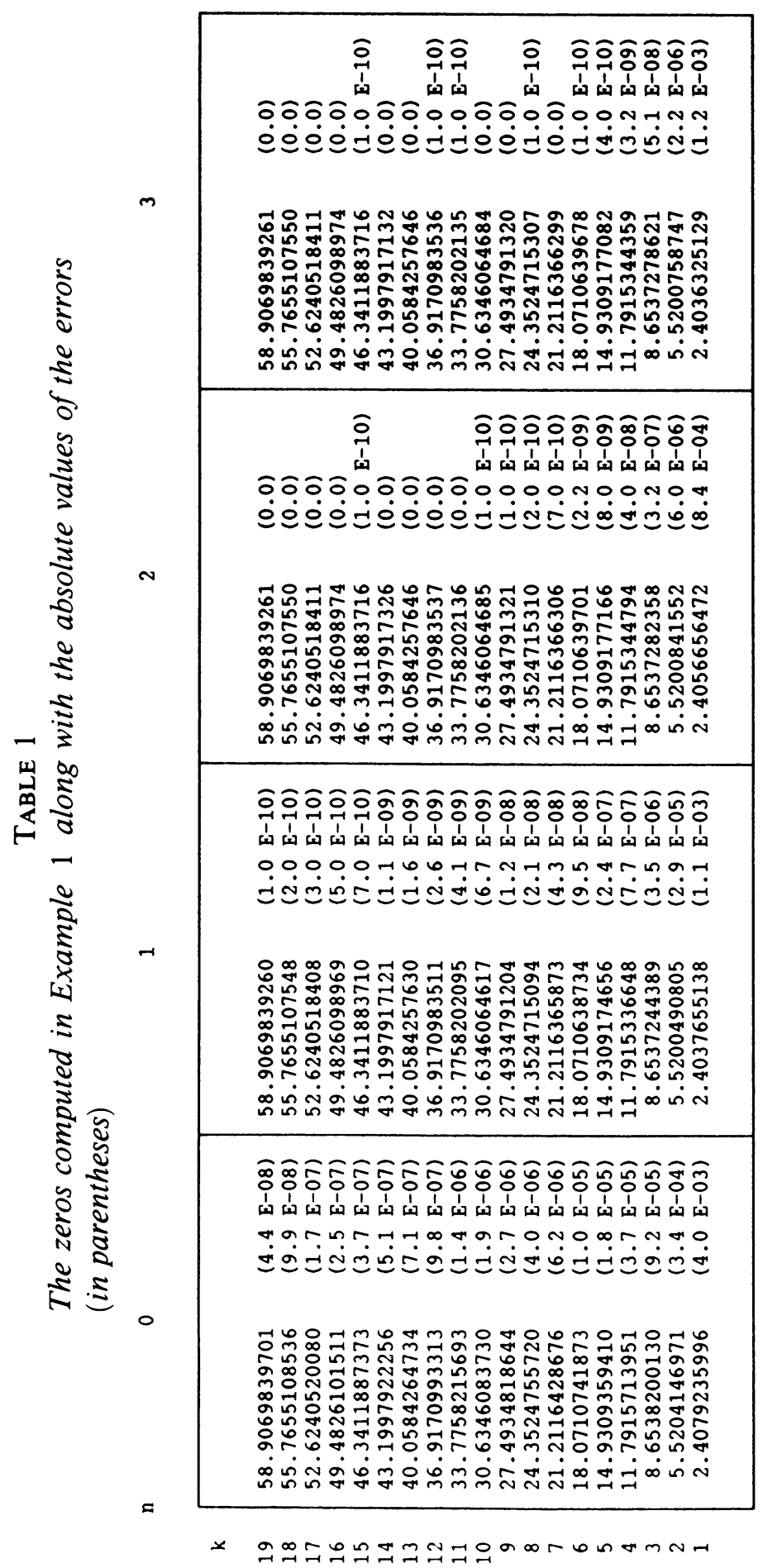




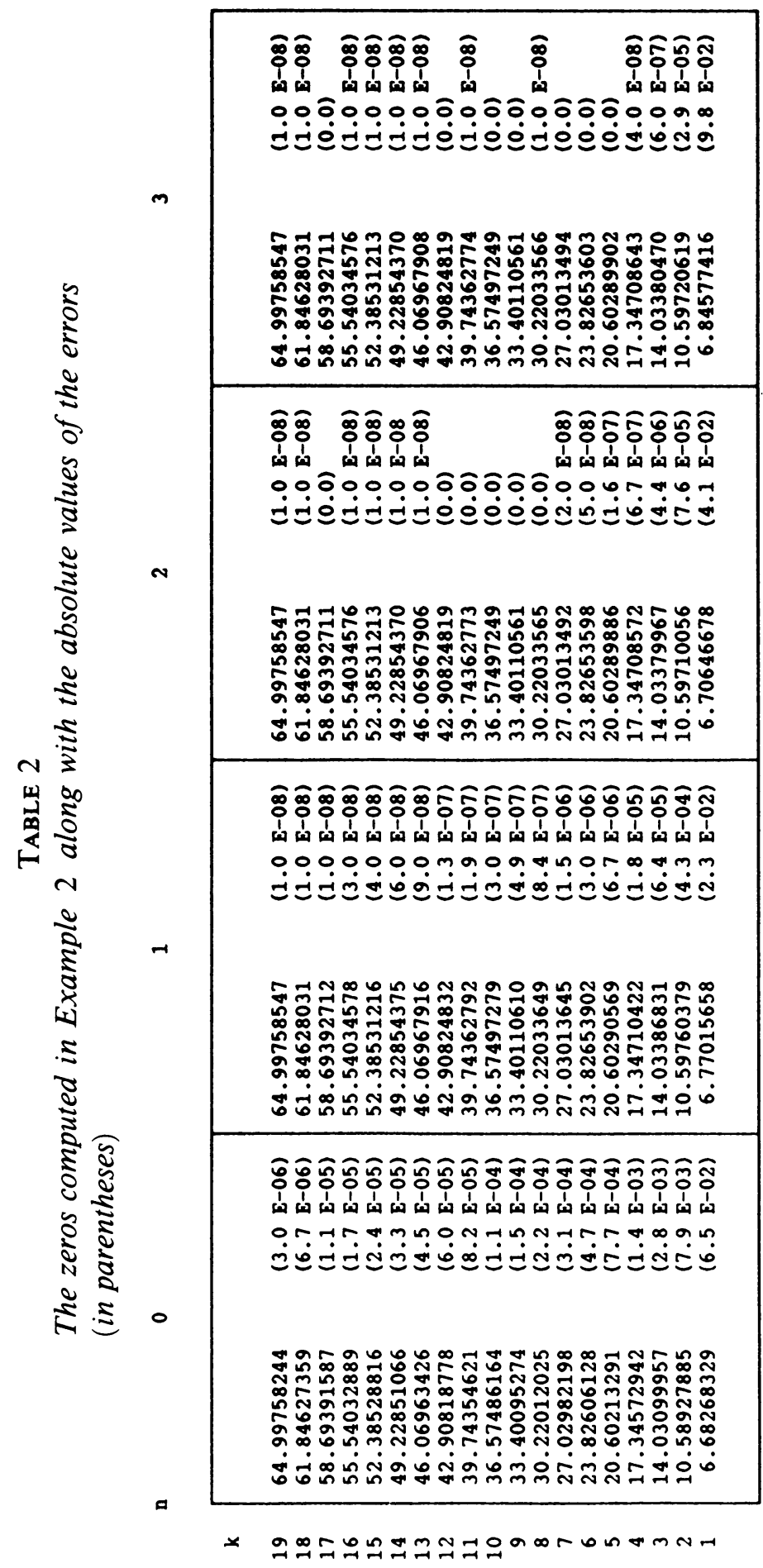




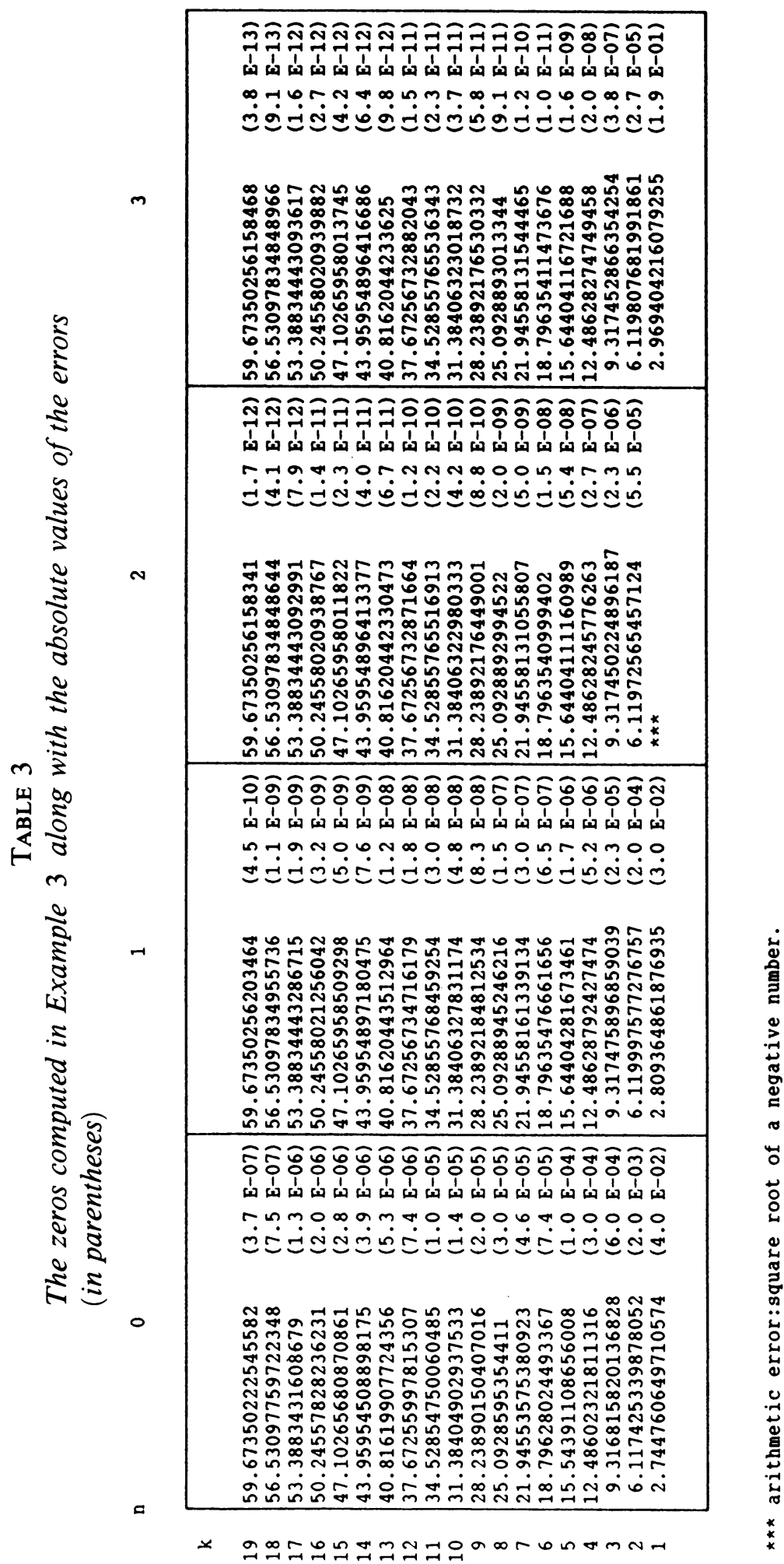




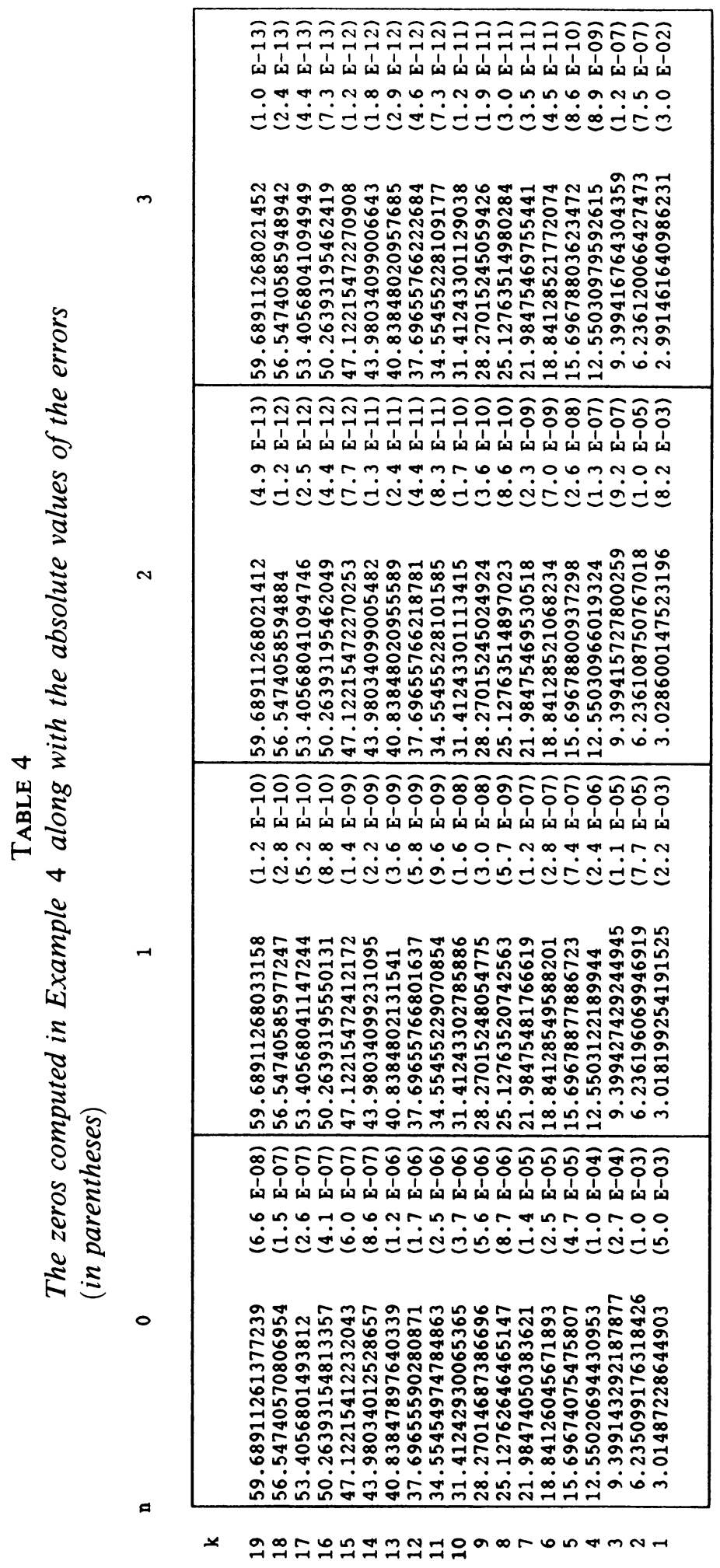


This is observable only for the first two or three zeros and can be ascribed to the fact that such zeros may lie outside of $S_{n}$. For example, the first zero in Examples 1, 3, and 4 lies certainly outside of $S_{2}$ and $S_{3}$, because $x>n \sqrt{2}$ for all $x \in S_{n} \cap \mathbf{R}$; cf. (3.15).

Remark 4.2. It is worth noting that the stationary points of every solution $y$ of (1.1), i.e., the zeros of $y^{\prime}(x)$, can be computed by approximating the socalled "second-phase" (cf. $[4, \S 5.8$, p. 39]). This can be done directly from the approximations of $\phi$; cf. [4, (5.34), p. 45].

\section{ACKNOWLEDGMENTS}

This work was carried out with financial support from the Italian National Council of Research (CNR). It was also supported by M.P.I., Numerical Analysis " $40 \%$ funds", and by M.P.I., Analysis " $60 \%$ funds". One of us (M.V.) is indebted to Professor R. Zanovello for continuous encouragement and advice.

\section{BIBLIOGRAPHY}

1. M. Abramowitz and I. A. Stegun (eds.), Handbook of mathematical functions, Dover, New York, 1968.

2. P. Appell, Sur la transformation des équations différentielles linéaires, C. R. Acad. Sci. Paris 91 (1880), 211-214.

3. R. Bellman, On the linear differential equations whose solutions are the products of the solutions of two given linear differential equations, Boll. Un. Mat. Ital. (3) 12 (1957), 12-15.

4. O. Boruvka, Linear differential transformations of the second order, English Univ. Press, London, 1971.

5. P. Hartman, Ordinary differential equations, Wiley, New York, 1964.

6. E. Hille, Lectures on ordinary differential equations, Addison-Wesley, New York, 1968.

7. S. Lang, Analysis. II, Addison-Wesley, Reading, Mass., 1969.

8. F. W. J. Olver, A new method for the evaluation of zeros of Bessel functions and of other solutions of second-order differential equations, Proc. Cambridge Philos. Soc. 46 (1950), 570-580.

9. __ (ed.), Bessel functions, Part III, Zeros and associated values, Roy. Soc. Math. Tables, vol. 7, Cambridge Univ. Press, Cambridge, 1960.

10. __ Asymptotics and special functions, Academic Press, New York, 1974.

11. R. Spigler, Alcuni risultati sugli zeri delle funzioni cilindriche e delle loro derivate, Rend. Sem. Mat. Univ. Politec. Torino 38 (1980), 67-85.

12. _ The linear differential equations whose solutions are the products of solutions of two given differential equations, J. Math. Anal. Appl. 98 (1984), 130-147.

13. F. G. Tricomi, Funzioni ipergeometriche confluenti, Consiglio Nazionale delle Ricerche, Monografie Mat., no. 1, Edizioni Cremonese, Roma, 1954.

Dipartimento di Metodi e Modelli Matematici per le Scienze Applicate, Università di Padova, via Belzoni 7, 35131 Padova, Italy

E-mail address: spigler@ipduniv.bitnet

Dipartimento di Matematica Pura e Applicata, Università di Padova, via Belzoni 7 , 35131 Padova, Italy 